\title{
Implementation of Fractal Image Compression on Medical Images by Different Approach
}

\author{
Mr. Vaibhav Vijay Bulkunde'1, Mr. Nilesh P. Bodne', Dr. Sunil Kumar² \\ 1Student, ${ }^{2}$ Professor \\ 1,2ECE Department, Kalinga University, Raipur, Chhattisgarh, India
}

\begin{abstract}
How to cite this paper: Mr. Vaibhav Vijay Bulkunde | Mr. Nilesh P. Bodne | Dr. Sunil Kumar "Implementation of Fractal Image Compression on Medical Images by Different Approach" Published in International Journal of Trend in Scientific Research and Development

(ijtsrd), ISSN: 2456-

6470, Volume-3 |

Issue-4, June 2019,

pp.398-400, URL:

https://www.ijtsrd.c

om/papers/ijtsrd23

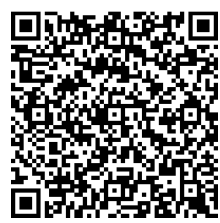

IITSRD23768
\end{abstract}

768.pdf

Copyright (C) 2019 by author(s) and International Journal of Trend in Scientific Research and Development Journal. This is an Open Access article distributed under the terms of the Creative Commons

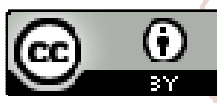

Attribution License (CC BY 4.0) by $/ 4.0$ ) (http://creativecommons.org/licenses/

ABSTRACT

FIC (Fractal Image Processing) is actually a JPG image which needs to be perform large scale encoding to improve and increase the compression ratio. In this paper we are going to analyze different constraints and algorithms of image processing in MATLAB so that there will be very low loss in image quality. It has been seen that whenever we contain any HD picture that to in medical application we need to sharpen the image that is we need to perform image encryption, image denoising and there should be no loss in image quality. In this paper, we actualized both consecutive and parallel adaptation of fractal picture pressure calculations utilizing programming show for parallelizing the program in Graphics Processing Unit for medicinal pictures, as they are very comparable inside the picture itself. Whenever we consider an image into fractal image, it has great importance and application in image processing field. In this paper compression scheme is used to sharpen and smoothen of image by using various image processing algorithm.

There are a few enhancements in the usage of the calculation too. Fractal picture pressure is based on the self-closeness of a picture, which means a picture having closeness in dominant part of the locales. We accept this open door to execute the pressure calculation and screen the impact of it utilizing both parallel and successive execution. Fractal pressure has the property of high pressure rate and the dimensionless plan. Pressure plot for fractal picture is of two kind, one is encoding and another is deciphering. Encoding is especially computational costly. Then again interpreting is less computational. The use of fractal pressure to restorative pictures would permit acquiring a lot higher pressure proportions. While the fractal amplification an indivisible element of the fractal pressure would be helpful in showing the recreated picture in an exceedingly meaningful structure. Be that as it may, similar to all irreversible strategies, the fractal pressure is associated with the issue of data misfortune, which is particularly troublesome in the therapeutic imaging. A very tedious encoding process, which can last even a few hours, is another troublesome downside of the fractal pressure.

Keywords: FIC (Fractal Image Processing), sharpening, smoothing, image compression

\section{INTRODUCTION}

Fractal Image Compression (FIC) is actually an idea in which the image is divided into sub-blocks, to do fractal compression. A resolution independent of any image of size $128 \times 128$ has to be analyze. The coding, storage and reconstruction of images remains an important factor in the application of computer technique to technical and scientific problem. Our research has focus mainly on the development of fractal method for compressing image data. This method is loss free and hence high compression ratio. Our method are computationally intensive but are feasible for non-real time application on computers. The image compression technique which we are going to use in this paper has purpose of increasing compression rate and computational time. Research on image processing present number of challenging field. One amongst them is image compression. A common characteristic of most images is that they are prone to computer redundancy. A number of diverse compression technique from lossy compression to lossless compression is encountered.

Fractal Image Compression (FIC) is relatively recent image compression method which are used for different part of image. The FIC algorithm initially begins with the complete image and partition the image into number of smaller blocks. Fractal compression uses encoding and decoding technique also. As we know enormous amount of therapeutic pictures are created regular and those are to be put away in some spot for future reference and utilization. It requires a great deal stockpiling and investment to store them. In this manner we demonstrate that on the off chance that we use GPUs to process the picture what's more, diminish the size for putting away, it bodes well. There may a few inquiries emerge that for what reason to consider restorative pictures as it were. The fundamental explanation for this is medicinal 
pictures are self-comparative furthermore, can be compacted effectively with better size decrease [18]. It is portrayed in more detail in the later segments. Then again, pictures with the exception of therapeutic zones, the greater part of them are not highly contrasting. They are mind boggling in nature which means the picture contain more data contrasted with highly contrasting. Along these lines it isn't useful to pack this kind of pictures. We won't get much decrease of the size after pressure as we have to put a large portion of the data flawless to get back the first picture [19]. Fractal picture pressure [2] technique will encode a picture as a lot of changes that are extremely comparable to the duplicate machine plot. For instance, the branch has detail at each level, the figure remade from the changes as well. The picture can be decoded at any size since it has no typical range. The extra component required for unraveling at bigger scale is generated involuntarily by the encoding transforms. We could decode an image of an individual escalating the size with each iteration, and ultimately can observe skin cells or perhaps atoms; it may be surprising if this detail is real. The response is in fact no. At what time the image was digitized, the detail is not at all associated to the actual detail present; it is just the artifact of the encoding transforms which initially only encoded the large- scale features. Nonetheless, in some occasion the detail is pragmatic at low magnifications, and this can be handy in Security and Medical imaging applications. As we know that the compression is based on finding the similar fragment and encoding them accordingly, thus there is redundant processing involved. So, the processing time becomes less if these redundant processing can be deployed to number of processors. This can be done with the help of parallel graphics processors. Hence, CUDA comes in the way to perform parallel processing. The remainder of this paper is organized as follows. In section II, we mentioned a concise overview of the related work done in this arena. We explained fractal image compression briefly in section III. In section IV, mentioning how we prepared our images, CPU and GPU configuration. In section $\mathrm{V}$, we demonstrate.

\section{FRACTAL IMAGE COMPRESSION (FIC)}

A new compression technique recently developed to compress the image is Fractal Image Compression (FIC). In the recent years FIC in many applications is utilize not only in medical but also in remote sensing geographic scheme. Different algorithms has been proposed to implement Fractal Image Compression based on partitional function. In other words we can say that we have to calculate the matrix of any image in the metrics form and then we have to apply image processing algorithms in wavelet transform in image processing. Each algorithm follows the same step. The classification of the system is carried out with the help of wavelet 3D transform in image processing using MATLAB.

\section{PERFORMANCE ANALYSIS}

In this section we will aquise that things in which image compression technique is well suited within compression time and hence high performance in reduction of computational time. The FIC uses a gray level scale image of Lena of size $128 \times 128$ has been considered for training the network using back propagation algorithm. A domain pool is created of size $4 \times 4$ for the Lena image and the standard deviation with histogram for Lena image has been carried out with special collaboration classes. The computer simulation has been carried out on MATLAB environment on
Intel ${ }^{\circledR}$ Core $^{\mathrm{TM}}$ i5-2430M CPU @ $2.40 \mathrm{GHz}$ processor of 4.00GB installed memory(RAM).

The image compression technique used in this project is found to have low Signal to Noise (SNR) with high encoding time and low decoding time. This algorithm is well suited for any color image also of size $256 \times 256$.

\section{METHODOLOGY}

Two classes of digital images can be distinguished - analog and digital images. Both types fall into non-temporal multimedia type. Analog images are painted or created through photographic process. During this process, the image is captured by a camera on a film that becomes a negative. We have a positive when the film is developed - no processing is possible from this moment. When the photography is made on a transparent medium then we are dealing with a diapositive (slide). Analog images are characterized by continuous, smooth transition of tones. This means that between each two different points at the picture there is an infinite number of tonal values. It is possible to transform an analog image into digital. The digitization process is usually caused by a need of digital processing. The output of digitalization is a digital approximation of the input analog image - the analog image is replaced by a set of pixels (points organized in rows and columns) and every pixel has a fixed, discrete tone value. Therefore, the image is not a continuous tone of colors. The precision and accuracy of this transformation depends on the size of a pixel - the larger area of an analog image transformed into one pixel the less precise approximation.

A. The key issues in FIC are proper selection of partitioning schemes to partition the image into domain and range blocks, transformation techniques to map the domain blocks to range blocks and speed up approaches to lessen the encoding time.

B. The present work while addressing the above problem has developed some approaches to enhance the performance of FIC on medical images. In the current work initially feasibility/suitability of FIC to medical images is investigated using standard conventional fixed partitioning FIC algorithm and further some speed up approaches is applied on medical images to reduce the encoding time.

C. The novel architecture for FIC using quad tree based partitioning technique for medical images has been implemented, modeled using MATLAB

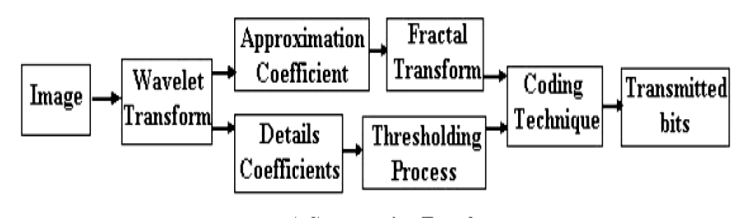

a) Compression Encoder

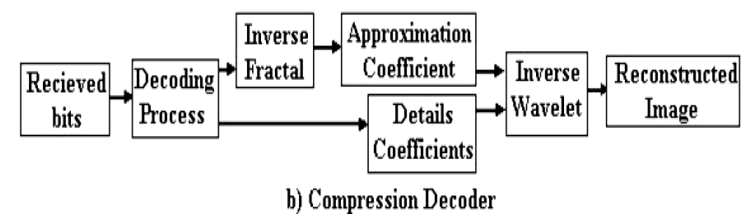

Fig: 1 Block Diagram of FIC

\section{CONCLUSION}

In this project we have done successful analysis of FIC method of medical sciences and the performances has been 
carried out. It is found that this technique is governed with single attributes and found out to be very efficient, lossless and high compression and low functionality of computational time.

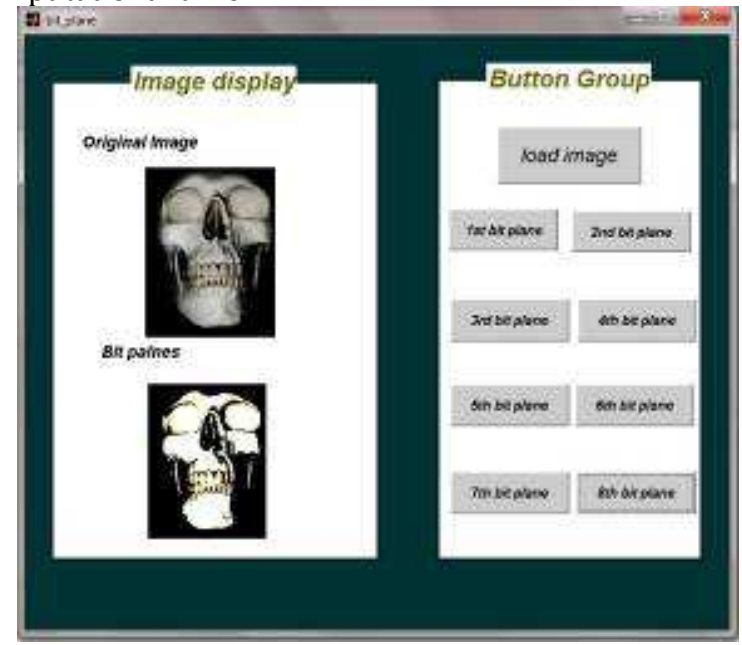

Fig: 2 Output before and after compression of image of a skull

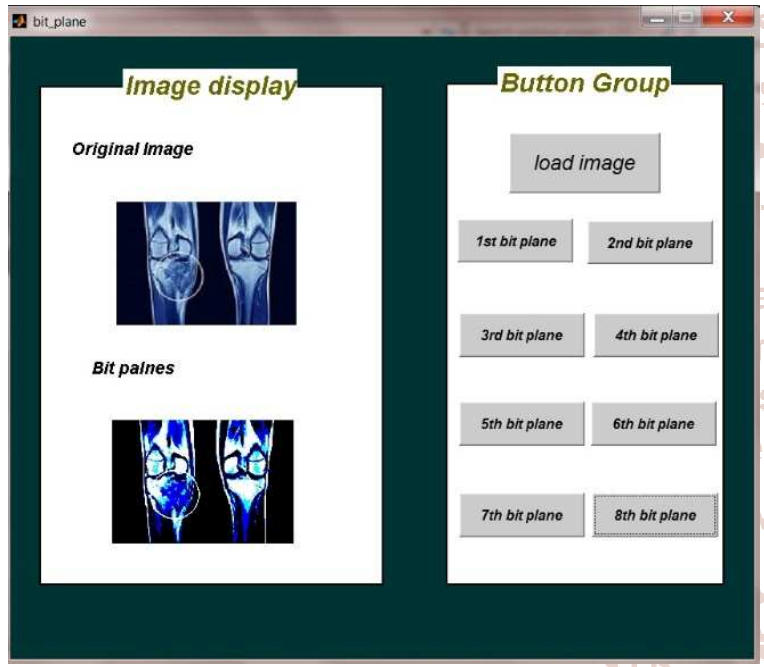

Fig: 3 Output before and after compression of image of a Xray of Leg

\section{REFRENCES}

[1] Mielikainen, Jarno, et al. Constant coefficients linear prediction for lossless compression of ultra-spectral sounder data using a graphics processing unit. Journal of Applied Remote Sensing 4.1 (2010): 041774041774 .

[2] M.F.Barnsley, L.P.Hurd andL.F.Anson,Fractal image compression. AK peters Wellesley, 1993.

[3] Wakatani, Akiyoshi. Improvement of adaptive fractal image coding on GPUs. Consumer Electronics (ICCE), 2012 IEEE International Conference on. IEEE, 2012.

[4] Franco, Joaqun, et al. A parallel implementation of the 2D wavelet transform using CUDA. Parallel, Distributed and Network-based Processing, 2009 17th Euromicro International Conference on. IEEE, 2009.
[5] Simek, Vaclav, and Ram Rakesh Asn. "GPU acceleration of $2 \mathrm{~d}$-dwt image compression in matlab with cuda." Computer Modeling and Simulation, 2008. EMS'08. Second UKSIM European Symposium on. IEEE, 2008.

[6] Lin, Caiwei, Lei Zhao, and Jiwen Yang. A High Performance Image Authentication Algorithm on GPU with CUDA. International Journal of Intelligent Systems and Applications (IJISA) 3.2 (2011): 52.

[7] Park, In Kyu, et al. Design and performance evaluation of image processing algorithms on GPUs. Parallel and Distributed Systems, IEEE Transactions on 22.1 (2011): 91-104.

[8] Monro, Donald M., and Frank Dudbridge. Fractal approximation of image blocks. Acoustics, Speech, and Signal Processing, 1992. ICASSP-92., 1992 IEEE International Conference on. Vol. 3. IEEE, 1992.

[9] Hutchinson, John E., and Ludger Rschendorf. Random fractal measures via the contraction method. Centre for Mathematics and its Applications, School of Mathematical Sciences, Australian National University, 1997.

[10] Marquardt, Donald W. An algorithm for least-squares estimation of nonlinear parameters. Journal of the Society for Industrial \& Applied Mathematics 11.2 (1963): 431-441.

[11] Oien, G. E., et al. A new improved collage theorem with applications to multi resolution fractal image coding. Acoustics, Speech, and Signal Processing, 1994. ICASSP94., 1994 IEEE International Conference on. IEEE, 1994.

[12] Falconer, Kenneth. Fractal geometry: mathematical foundations and applications. Wiley.com, 2007.

[13] - Mandelbrot, Benoit B., and A. Blumen. Fractal Geometry: What is it, and What Does it do? [and Discussion]. Proceedings of the Royal Society of London. A. Mathematical and Physical Sciences 423.1864 (1989): 3- 16.

[14] Dasgupta, Abhijit. Brouwers Theorem and Sierpinskis Theorem. Set Theory. Springer New York, 2014. 313319.

[15] Barnsley, Michael F., et al. Solution of an inverse problem for fractals and other sets. Proceedings of the National Academy of Sciences of the United States of America 83.7 (1986): 1975.

[16] Erra, Ugo. Toward real time fractal image compression using graphics hardware. Advances in Visual Computing. Springer Berlin Heidelberg, 2005. 723-728.

[17] Sodora, August. Fractal Image Compression on the Graphics Card. Hopkins Undergraduate Research Journal, Johns Hopkins University (2010).

[18] Che, Shuai, et al. A performance study of generalpurpose applications on graphics processors using CUDA. Journal of parallel and distributed computing 68.10 (2008): 1370-1380. 